\title{
Brasil: ¿Cómo reaccionan los mercados financieros ante los anuncios de política monetaria del banco central en un esquema de metas de inflación?
}

\author{
Gabriel Caldas $M$.
}

Los anuncios del banco central son trascendentales para guiar las expectativas. Empero, la investigación correspondiente con respecto a los países que emergen es escasa. Dado que el Brasil es un importante país emergente con políticas de fijación de objetivos de inflación, se analiza aquí la influencia de la política monetaria y los anuncios del banco central en la estructura temporal de las tasas de interés. Mediante el método de mínimos cuadrados ordinarios (MCO), el método generalizado de momentos (MGM) y el modelo de autorregresión vectorial (VAR) se examina la dirección de las tasas de interés al ser afectadas por los anuncios del banco central y la política monetaria. Puesto que los agentes económicos analizan las actas de las reuniones del Comité de Política Monetaria, la política y los anuncios mencionados influyen poderosamente en la formación de expectativas sobre las tasas de interés para diferentes plazos en el Brasil. investigador del Consejo Nacional de Desarrollo Científico y Tecnológico (CNPq). 


\section{I}

\section{Introducción}

Para mantener una inflación baja y estable se requieren políticas creíbles, comprometidas y transparentes que guíen las expectativas. Según Blinder y otros (2008), a medida que se hizo cada vez más evidente que el manejo de las esperanzas estadísticas es una parte útil de la política monetaria, la política de anuncios pasó de ser una molestia a un instrumento esencial entre las herramientas del banco central. El análisis de los anuncios de este banco es sobre todo importante en países emergentes con políticas de fijación de objetivos inflacionarios, dado que las incertidumbres acerca del control de la inflación en esos países (Mishkin y Savastano, 2001) hacen más difícil guiar las expectativas.

Según Blinder y otros (2008, pág. 940), los anuncios pueden ser una pieza relevante entre las herramientas con que cuenta el banco central, debido a su capacidad de provocar movimientos en los mercados financieros. No obstante, aún es necesario obtener evidencia empírica. Tal como señalan Blinder y otros (2008, pág. 941), en los pocos estudios en que se procura evaluar la intención direccional de los anuncios del banco central, se observa, en general, que los mercados reaccionan en la dirección "correcta"; es decir, aquello que anteriormente se conocía como "efectos de los anuncios" en lugar de desfavorecer al banco central, lo ayudan. Empero, hasta ahora el tema ha sido poco investigado. Se requiere obtener más evidencia para alcanzar la convicción en esta materia, además de un análisis más profundo de cómo, si tal fuera el caso, la creación de estos anuncios, que señalan una dirección, aumenta el bienestar.

La literatura relacionada con los países desarrollados es amplia y creciente ${ }^{1}$; en cambio, la investigación sobre los países emergentes es escasa. En el presente estudio

\footnotetext{
1 Por ejemplo, Kohn y Sack (2004); Connolly y Kohler (2004); Musard-Gies (2006); Andersson, Dillén y Sellin (2006); Rosa y Verga (2007); Reeves y Sawicki (2007); Ehrmann y Fratzscher (2007a y 2009); Hayo y Neuenkirch (2010); Brand, Buncic y Turunen (2010); Hayo, Kutan y Neuenkirch (2010); Ranaldo y Rossi (2010); Sturm y De Haan (2011); Rosa (2011); Berger, De Haan y Sturm (2011).
}

se analiza la política monetaria y los anuncios del banco central del Brasil. Dado que se trata de un importante país emergente con políticas de fijación de objetivos de inflación, y teniendo en cuenta la creciente relevancia de dichos anuncios, en este trabajo se analizan las actas de las reuniones del Comité de Política Monetaria (СОРOM) a fin de evaluar la influencia de la política monetaria y los anuncios del banco central en el tramo corto de la estructura temporal de las tasas de interés.

El trabajo contribuye a la literatura de las siguientes maneras: en primer término, a diferencia de los estudios anteriores sobre los anuncios del banco central, en la presente investigación se complementa el análisis mediante el método de mínimos cuadrados ordinarios (MCO) por medio del uso del método generalizado de momentos (MGM) y del modelo de autorregresión vectorial (VAR), con el propósito de analizar la dirección que toman las tasas de interés al verse afectadas por los anuncios del banco central y la política monetaria (en ningún estudio se utiliza el método VAR para analizar en forma dinámica si los anuncios del banco central influyen en la dirección de las tasas de interés). En segundo lugar, en el estudio se proporciona un análisis respecto de la estructura temporal de un importante país emergente con políticas de fijación de objetivos de inflación, sobre la base de las ideas dadas a conocer en Fuhrer y Moore (1995a y 1995b); Fuhrer (1996) y Walsh (2010). El tipo de análisis presentado en este trabajo no se ha llevado a cabo en ningún otro estudio sobre los anuncios del banco central.

El presente trabajo se organiza según se describe a continuación. En la sección II se ofrece un breve resumen de la literatura sobre política monetaria y anuncio de medidas adoptadas por el banco central. En la sección III se utilizan las actas de las reuniones del COPOM para examinar la repercusión de la política monetaria y los anuncios del banco central en el tramo corto de la estructura temporal de las tasas de interés en el Brasil, y también se analizan los resultados empíricos. Las conclusiones se presentan en la sección IV. 


\section{II}

\section{Los anuncios del banco central y la política monetaria. Revisión de la literatura}

A principios de la década de 1990, la fijación de objetivos de inflación se convirtió en una práctica frecuente, lo que acrecentó la conciencia respecto de la importancia de la transparencia en el proceso de formación de expectativas (Geraats, 2002; De Mendonça y Simão-Filho, 2007). En un contexto prospectivo, la política monetaria se convierte en gran medida en el arte de manejar las expectativas de los agentes privados (Woodford, 2003). En consecuencia, los estudios sobre los anuncios del banco central han adquirido relevancia en la literatura relativa a este régimen.

El grado de transparencia y la regularidad del proceso de comunicación dependen del nivel de discreción existente en el régimen monetario adoptado. Por lo tanto, la complejidad de la comunicación se relaciona con el tipo de régimen monetario puesto en práctica. Los regímenes con menos discreción se relacionan con una comunicación más simple, mientras que los esquemas más flexibles y que permiten mayor discreción, tales como la fijación de objetivos de inflación, suponen una comunicación más compleja (Blinder y otros, 2008).

Los bancos centrales que fijan objetivos de inflación, en general, efectúan anuncios con relación a cuatro aspectos de la política monetaria: sus objetivos y estrategias, las razones que hay detrás de las decisiones políticas, las perspectivas sobre el estado de la economía y las decisiones futuras en materia de política monetaria. Puesto que los objetivos y las estrategias de los bancos centrales tienden a ser estables $\mathrm{y}$, en consecuencia, varían menos a lo largo del tiempo que los otros tres aspectos, los anuncios con relación a este aspecto son menos frecuentes.

En la literatura se incluyen dos enfoques principales, que son complementarios, para analizar la influencia de las comunicaciones en la economía. En uno se explora el efecto de los anuncios del banco central en los mercados financieros (por ejemplo, Kohn y Sack, 2004; Reeves y Sawicki, 2007; Ehrmann y Fratzscher, 2007a y 2009), mientras que el otro se enfoca en la influencia de las distintas estrategias de comunicación de los bancos centrales en el desempeño de la economía (por ejemplo, Fujiwara, 2005; Rozkrut y otros, 2007). Dado que los anuncios del banco central influyen en las expectativas con respecto a la tasa de interés futura a corto plazo, también afectan a las tasas de interés a largo plazo y, por lo tanto, a los precios del mercado financiero. A su vez, estos precios pueden afectar a algunas variables macroeconómicas, como la inflación y el producto. No obstante, mientras que los anuncios y la política monetaria afectan instantáneamente al mercado financiero, la economía en conjunto se ve afectada en forma gradual $\mathrm{y}$ por varios otros factores.

Existen básicamente tres enfoques para medir el alcance de los anuncios del banco central. En el primero se procura clasificar todos los anuncios del banco según su contenido o señales (posibles intenciones), mediante una escala numérica para codificar esas clasificaciones (Jansen y De Haan, 2005; Ehrmann y Fratzscher, 2007b; Rosa y Verga, 2007; Musard-Gies, 2006). En algunos estudios se utilizan los códigos para indicar una dirección (Jansen y De Haan, 2005; Ehrmann y Fratzscher, 2007b). En el segundo enfoque se analizan todas las formas de comunicación mediática del copom o de sus miembros que resulten de relevancia para dicha política (Jansen y De Haan, 2005; Ehrmann y Fratzscher, 2007b). En este caso, la tarea es extraer información de las declaraciones, los discursos, las actas y los informes mediante el uso de un conjunto de palabras de búsqueda. Cuando el objetivo es evaluar la tendencia de la política monetaria, en la búsqueda se incluye el nombre del miembro del COPOM y las palabras clave "tasa de interés", "política monetaria" e "inflación". Cuando el objetivo es evaluar la situación económica futura, las palabras clave son "economía” y "perspectivas económicas". Por último, en el tercer enfoque se aprovechan algunas características institucionales del proceso de anunciar las decisiones de política monetaria para medir la repercusión de los anuncios. Un ejemplo de este enfoque estaría dado por Brand, Buncic y Turunen (2010), quienes dividen las reacciones del mercado financiero en dos efectos: el efecto de la política monetaria y el de los anuncios del Banco Central Europeo (BCE).

En la literatura se han desarrollado diferentes enfoques destinados a medir los efectos de los anuncios del banco central (para mayor información, véase Blinder y otros, 2008). Comenzando por Kohn y Sack (2004), en 
algunos estudios se han examinado los efectos de dichos anuncios en la volatilidad de las variables financieras (Blinder y otros, 2008, presentan varios de estos estudios). El foco en la volatilidad hace que sea innecesario asignar una dirección a cada declaración. En otros estudios, los anuncios se cuantifican a objeto de evaluar tanto la dirección (por ejemplo, Ehrmann y Fratzscher, 2007b) como la magnitud (por ejemplo, Berger, De Haan y Sturm, 2011) de los efectos en los precios de los activos, y de ese modo poder establecer la medida en que un anuncio determinado logra el efecto deseado.

En años recientes, la tendencia general de los bancos centrales a favorecer la transparencia, la rendición de cuentas y la comunicación ha motivado una serie de estudios sobre la influencia de los anuncios de los bancos centrales en los mercados financieros ${ }^{2}$. Sin embargo, esta literatura está dirigida principalmente a los países desarrollados. Para el Brasil, Costa Filho

2 Por ejemplo, Kohn y Sack (2004); Connolly y Kohler (2004); MusardGies (2006); Andersson, Dillén y Sellin (2006); Rosa y Verga (2007); Reeves y Sawicki (2007); Ehrmann y Fratzscher (2007a y 2009); Brand, Buncic y Turunen (2010); Berger, De Haan y Sturm (2011). y Rocha (2009) analizan el papel de los anuncios en la conducción de la política monetaria por parte del Banco Central del Brasil. Ellos desarrollan un glosario que traduce la información cualitativa contenida en las actas del сором a un índice de escala ordenado, similar al elaborado por Rosa y Verga (2007). Según estos autores, hay evidencia de un comportamiento coherente por parte del COPOM, en el sentido de que sus palabras son seguidas de acciones en la misma dirección. Asimismo, sobre la base de estimaciones de reglas de Taylor, también encuentran evidencia de que el índice ayuda a comprender la fijación de la tasa de interés. En un trabajo posterior, Costa Filho y Rocha (2010) analizan si una mejor comunicación por parte del Banco Central del Brasil haría más predecible la política monetaria. Los autores observaron que las tasas de interés aumentan en los días de publicación, lo que indica que los anuncios del banco central tienen un sesgo conservador, al tiempo que disminuye la volatilidad de las tasas de interés. También encontraron evidencia de que la reacción del mercado es independiente del contenido publicado, aunque las señales de una baja de las tasas de interés implican una reducción de la volatilidad.

\section{III}

\section{Los anuncios del Banco Central del Brasil}

En 1999 se fijaron objetivos de inflación en el Brasil. Desde entonces, la conducción de la política monetaria por parte del banco central del país se ha vuelto más transparente y responsable y los anuncios han sido más frecuentes y abiertos, a medida que los encargados de formular las políticas comprendieron la relevancia que tienen las expectativas en la economía. Este cambio coincide con la tendencia de los bancos centrales más importantes del mundo, en especial aquellos que fijan objetivos de inflación.

En el presente trabajo se utiliza la información contenida en las actas de las reuniones del сором para analizar el caso brasileño. Las reuniones ordinarias del COPOM se desarrollan durante dos días, la primera sesión tiene lugar los martes y la segunda, los miércoles. El primer día de la reunión, los jefes de departamento presentan un panorama de la coyuntura económica. El segundo día, los directores de política monetaria y política económica presentan alternativas para la tasa de interés a corto plazo y sus recomendaciones en materia de política monetaria.
Otros miembros del COPOM presentan sus comentarios y propuestas, y luego se procede a la votación sobre la base de una propuesta final con el objeto de alcanzar un consenso. Posteriormente, se anuncia la decisión final: el objetivo para la tasa de interés del Sistema Especial de Liquidación y Custodia (SELIC) y el sesgo, si lo hubiera.

Ocho días después de cada reunión, el сором publica las actas correspondientes en el sitio web del banco central, donde se proporciona un resumen de las discusiones del Comité. En el documento se describe el estado de la economía, qué factores fueron relevantes para la decisión tomada en la reunión y las perspectivas para las reuniones futuras.

Sobre la base de la información disponible, los agentes intentan estimar el estado de la economía a largo plazo y el comportamiento futuro de los encargados de formular la política monetaria. En este sentido, la política monetaria y los anuncios del сором por medio de las actas serán efectivas en tanto logren guiar las expectativas de los agentes. 


\section{Cálculo}

En esta subsección se calculan los efectos de los anuncios del banco central en el tramo corto de la estructura temporal de las tasas de interés en el Brasil. Las anuncios se cuantifican por medio de variables ficticias (dummy) y el diferencial de tasas de interés es la diferencia entre una tasa de interés con un plazo específico de hasta un año (medida por una tasa de intercambio swap) y la tasa básica de interés (medida por la tasa SELIC) ${ }^{3}$.

Con tal fin, en el análisis se utilizaron los siguientes datos: la tasa swap de referencia a 360 días, sobre la base de la tasa anual de interés interbancaria prefijada de la Bolsa de Mercaderías y Futuros del Brasil (BM\&F) ${ }^{4}$; la tasa swap anual de referencia a 180 días, basada en la

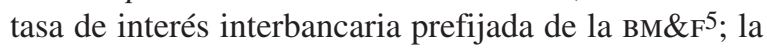
tasa swap anual de referencia a 120 días, basada en la tasa de interés interbancaria prefijada de la BM\&F en porcentaje anual6; y la tasa de interés SELIC anualizada, acumulada en el mes ${ }^{7}$.

El período de análisis se extiende de junio de 2003 a abril de 2011, y comprende desde la $85^{\text {a }}$ reunión hasta la $158^{a}$ reunión del COPOM (74 observaciones). Se decidió comenzar con la $85^{\mathrm{a}}$ reunión dado que hasta esta no había una regla clara sobre la difusión de las actas de las reuniones anteriores. En consecuencia, las actas han sido publicadas en forma periódica recién a partir de la $85^{\text {a }}$ reunión.

Las variables ficticias se construyen sobre la base de un glosario preparado (véase el cuadro A.1 del apéndice), diseñado para codificar las palabras y expresiones contenidas en las actas del COPOM. El glosario se inspira en el trabajo de Rosa y Verga (2007). Cuando hay una indicación de que el COPOM tiene previsto reducir la tasa SELIC en su próxima reunión, la variable ficticia dummy_red toma un valor de 1 (y de 0 en cualquier otro caso). Cuando hay una indicación de que el copom no cambiará la tasa SELIC en su próxima reunión, la variable ficticia dummy_neutra toma un valor de 1 (y de 0 en cualquier otro caso). Cuando hay una indicación de que el COPOM tiene previsto aumentar la tasa SELIC en su

\footnotetext{
${ }^{3}$ La elección de tasas swap como medida de las tasas de interés de distintos plazos está en concordancia con la literatura sobre la estructura temporal de las tasas de interés en la economía brasileña. Por ejemplo, Lima e Issler (2003); Tabak y Tabata (2004); y Montes y Bastos (2011) utilizan las mismas tasas swap como variables sustitutivas de las tasas de interés a largo plazo y del diferencial de tasas de interés.

${ }^{4}$ Código 7806 (sitio web del Banco Central del Brasil).

${ }^{5}$ Código 7805 (sitio web del Banco Central del Brasil).

${ }^{6}$ Código 7804 (sitio web del Banco Central del Brasil).

${ }^{7}$ Código 4189 (sitio web del Banco Central del Brasil).
}

próxima reunión, la variable ficticia dummy_aum toma un valor de 1 (y de 0 en cualquier otro caso).

A fin de analizar la repercusión de estos anuncios en el tramo corto de la estructura temporal de las tasas de interés en el Brasil, se calcularon tres ecuaciones sobre la base de la siguiente ecuación básica8:

$$
\begin{gathered}
\text { Diferencial }_{k t}=\beta_{1 k} \text { dummy_aum }_{t}+ \\
\beta_{2 k} \text { dummy_neutra }_{t}+\beta_{3 k} \text { dummy_red }_{t}+\zeta_{t k}
\end{gathered}
$$

donde el Diferencial ${ }_{k t}$ es la diferencia entre la tasa swap a un plazo $k(k=120,180,360)$ y la tasa básica de interés (SELIC) vigente en la fecha $t$, y $\zeta_{t k}$ representa los términos de error (ruido blanco). Las series de Swap_360,Swap_180 y Swap_120 se basaron en la información del día posterior a la publicación de las actas del сором en el sitio web del Banco Central del Brasil. Los resultados esperados son $\beta_{1 k}>0, \beta_{2 k}=0$ (es decir, no significativos) y $\beta_{3 k}<0$.

Los anuncios del COPOM serán efectivos en la medida en que las expectativas sobre las tasas de interés se comporten de acuerdo con la señal dada por la autoridad monetaria, según lo identificado en las actas. La idea es verificar si la política monetaria y los anuncios sirven como guía de las expectativas sobre las tasas de interés y, en consecuencia, afectan al tramo corto de la estructura temporal de las tasas de interés en el Brasil en la dirección deseada. El análisis se llevó a cabo por medio de MCO y el modelo VAR.

En el cuadro 1 se muestra que los coeficientes estimados para dos de las variables ficticias (dummy_aum y dummy_red) son significativos para todos los plazos y presentan los signos esperados. Los coeficientes estimados para la variable ficticia neutral (dummy_neutra) no son estadísticamente significativos, lo que significa que cuando el сором señala que no cambiará la tasa SELIC los diferenciales no cambian.

La evidencia presentada hasta ahora ha demostrado que cuando el banco central indica que ajustará la política monetaria, los diferenciales reaccionan aumentando, y cuando el banco señala una relajación, los diferenciales disminuyen. Una cuestión importante que surge es la influencia que ejercen la política monetaria y los anuncios del banco central en las tasas a corto plazo, de manera dinámica. Los modelos de autorregresión vectorial (VAR), en general, emplean funciones de impulso-respuesta, dado que estas permiten evaluar el

\footnotetext{
${ }^{8}$ Las ecuaciones se calcularon sin intersección para evitar la multicolinealidad perfecta.
} 
CUADRO 1

Efecto de las variables ficticias sobre los diferenciales

\begin{tabular}{lccc}
\hline \multirow{2}{*}{ Variables explicativas } & \multicolumn{2}{c}{ Variables dependientes } & Diferencial $_{360}$ \\
\cline { 2 - 4 } & Diferencial $_{120}$ & Diferencial $_{180}$ & $0,91^{* * * *}$ \\
dummy_aum & $0,45^{* * * *}$ & $0,65^{* * *}$ & $(0,00)$ \\
& $(0,00)$ & & $0,00)$ \\
dummy_neutra & $-0,013$ & $(0,94)$ & 0,35 \\
& $(0,89)$ & $-0,95^{* * *}$ & $(0,10)$ \\
dummy_red & $-0,75^{* * *}$ & $(0,00)$ & $-1,14 * * *$ \\
Estadísticas resumidas & $(0,00)$ & $0,00)$ & \\
$R^{2}$ & & 0,55 & 0,45 \\
$R^{2}$ ajustado & 0,54 & 0,53 & 0,43 \\
Error estándar & 0,52 & 0,62 & 1,01 \\
Número de observaciones & 0,48 & 74 & 74 \\
\hline
\end{tabular}

Fuente: cálculos del autor.

Notas: las ecuaciones se calculan utilizando MCO; los valores $p$ están entre paréntesis.

*** Nivel de significancia estadística del $1 \%$.

impacto de shocks (o innovaciones) en variables clave a lo largo del tiempo (Sims, 1980). Tal como plantea Lutkenpohl (1991), con el método convencional se aplica un supuesto de ortogonalidad de manera tal que el resultado puede depender del ordenamiento de las variables en el modelo VAR. Koop, Pesaran y Potter (1996); y Pesaran y Shin (1998) desarrollan la idea de la función de impulso-respuesta generalizada como forma de eliminar el problema del ordenamiento de las variables en el modelo VAR. El argumento principal es que las funciones de impulso-respuesta generalizadas no varían ante un reordenamiento de las variables en el modelo VAR (véase, por ejemplo, Ewing, 2003).

En el análisis se utiliza la tasa SELIC, Swap_360, Swap_180, Swap_120,dummy_red y dummy_aum. Dado que las distintas series para las variables Swap_360, Swap_180 y $S w a p_{-120}$ presentan quiebres estructurales en las medias (como muestran los gráficos A.1, A.2 y A.3 del apéndice), el modelo VAR incluye una variable ficticia (denominada dummy_break) como variable exógena, donde la variable dummy_break toma un valor de uno (1) desde la $85^{\mathrm{a}}$ reunión hasta la $123^{\mathrm{a}}$ reunión, y de cero (0) en cualquier otro caso.

Para verificar si las series son estacionarias, es decir, si la series no tienen una raíz unitaria, se aplicaron las pruebas de Kwiatkowski-Phillips-Schmidt-Shin (KPSS) y Phillips-Perron (PP) (véanse los cuadros A.2 y A.3 del apéndice). Se utilizaron los criterios de información de Akaike, Schwarz y Hannan-Quinn para seleccionar el orden del VAR. Los resultados indican que el orden del
VAR es uno (1) (véase el cuadro A.4 del apéndice). En el gráfico 1 se aprecia la estabilidad del vAR estimado.

En el gráfico 2 se presentan los resultados de las funciones de impulso-respuesta generalizadas para un horizonte temporal de 18 meses. La evidencia indica que cuando las actas del COРOM señalan un incremento en la tasa de interés SELIC para la próxima reunión, las tasas de interés reaccionan primero aumentando y después convergiendo al punto de equilibrio. Cuando

GRÁFICO

\section{Estabilidad del modelo VAR}

Raíces inversas del polinomio característico AR

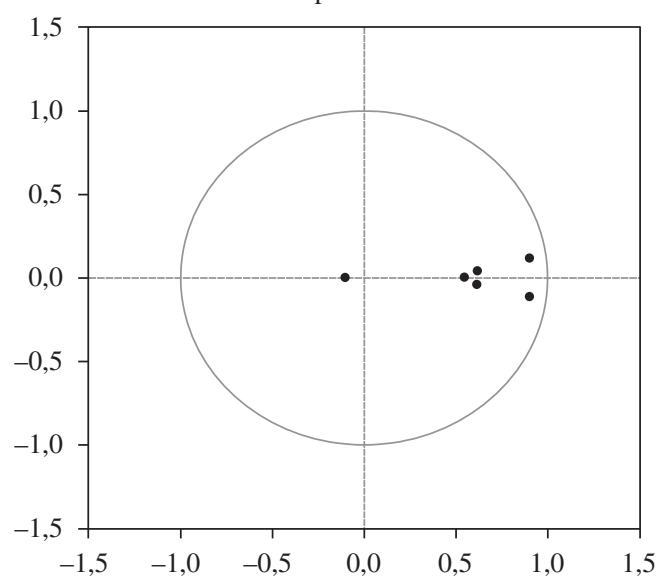

Fuente: elaboración propia. 
GRÁFICO 2

\section{Impulso-respuesta a shocks de una desviación estándar}

( \pm 2 desviaciones estándar)
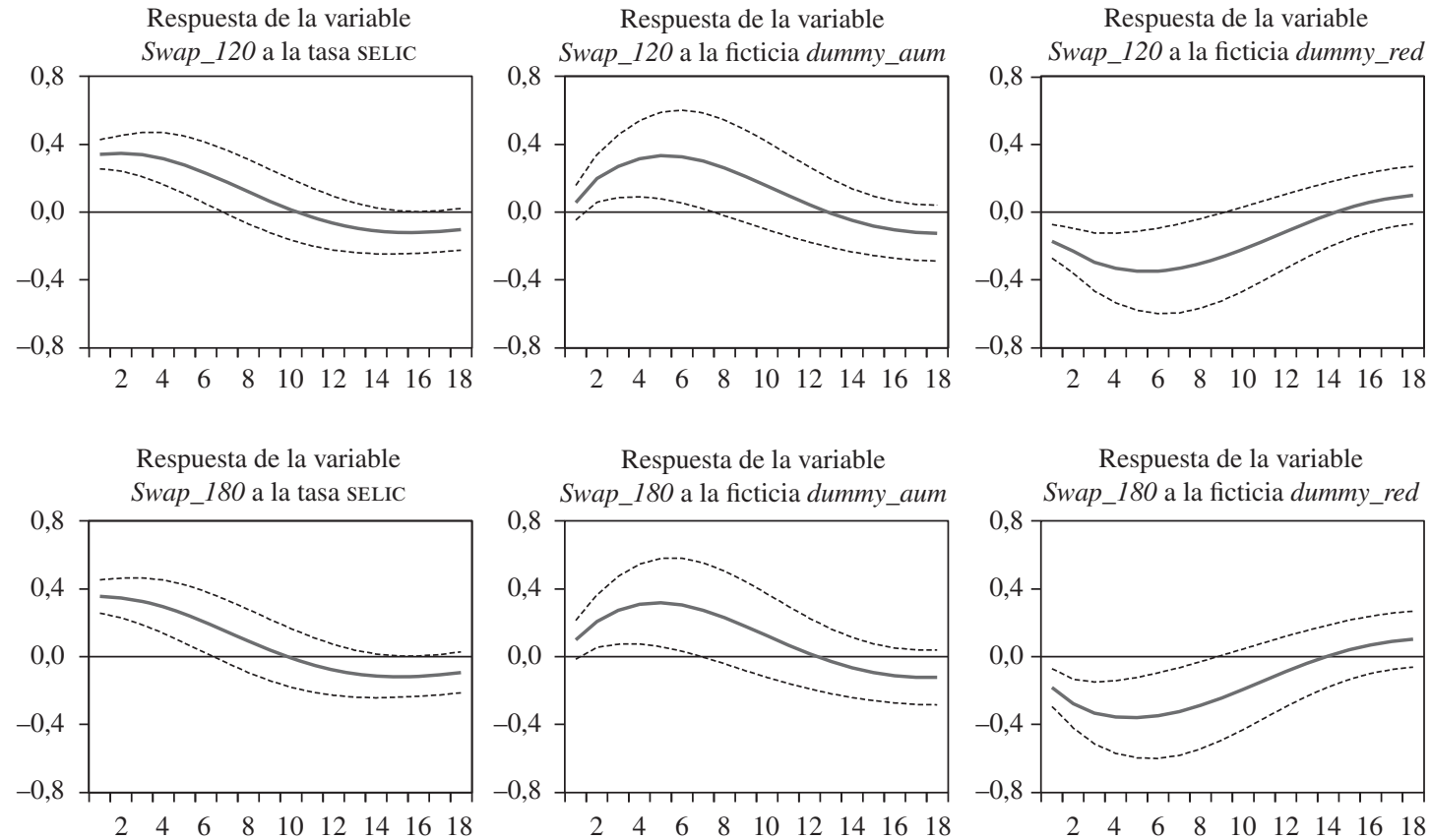

Respuesta de la variable Swap_360 a la tasa SELIC

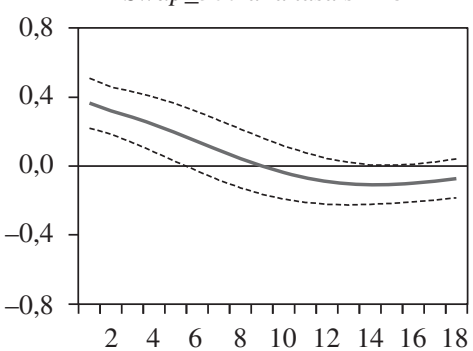

Respuesta de la variable Swap_360 a la ficticia dummy_aum

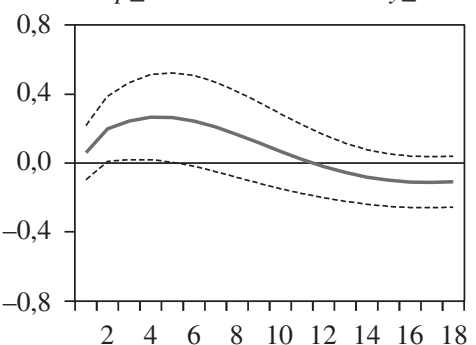

Respuesta de la variable Swap_360 a la ficticia dummy_red

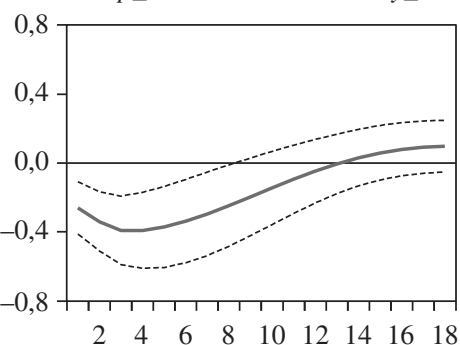

Fuente: elaboración propia.

en el сором se indica una reducción de la tasa de interés SELIC para la próxima reunión, las tasas de interés reaccionan disminuyendo y luego convergiendo al equilibrio. Estos hallazgos son estadísticamente significativos. Con respecto a la influencia de los shocks de política monetaria en el tramo corto de la estructura temporal de las tasas de interés, los resultados sugieren que los shocks positivos en la tasa de interés SELIC provocan un aumento de las tasas de interés. Estos resultados son también estadísticamente significativos.
La evidencia obtenida hasta ahora sugiere que la política monetaria y los anuncios del COPOM juegan un papel relevante en el proceso de formación de expectativas sobre el comportamiento de las tasas de interés en el Brasil. Dado que las tasas de interés a largo plazo también reflejan aspectos macroeconómicos, se procederá a analizar la influencia de la política monetaria y los anuncios del соРом en el tramo corto de la estructura temporal de las tasas de interés, así como la influencia de las expectativas de inflación y las desviaciones del producto respecto de su tendencia. Con este fin se 
agregaron las siguientes series: desviaciones del producto (la diferencia entre el producto interno bruto (PIB) ${ }^{9}$ y el PIB de tendencia) ${ }^{10}$ y las expectativas de inflación (expectativas del mercado con respecto a la inflación en los próximos 12 meses, medida según el Índice Nacional de Precios al Consumidor Amplio (IPCA) ${ }^{11}$.

A continuación, se presenta una ecuación basada en Fuhrer y Moore (1995a y 1995b), Fuhrer (1996) y Walsh (2010), con el objetivo de analizar la influencia de la política monetaria, los anuncios del banco central, las desviaciones del producto y las expectativas de inflación en las tasas a corto plazo:

$$
\begin{gathered}
S_{k t}=\alpha_{k} \pi_{t-1}^{e}+\delta_{k}\left(y_{t-1}-y_{t-1}^{*}\right)+ \\
\gamma_{k}\left(r_{t-1}\right)+\text { anuncios }_{t}+\varepsilon_{k t}
\end{gathered}
$$

donde $S$ es la tasa swap con un plazo $k(k=120,180$, $360), \pi^{e}$ representa las expectativas de inflación (con una anticipación de 12 meses), ( $\left.y-y^{*}\right)$ representa las desviaciones del producto respecto de la tendencia (que es una medida del ciclo económico), $r$ es la tasa básica de interés (SELIC) y $\varepsilon$ es el término de error. Las variables macroeconómicas se utilizaron con un retardo, dado que este es el conjunto de información disponible para los agentes económicos en el momento en que estos forman sus expectativas en relación con las tasas de interés. Los signos esperados para los coeficientes de las variables macroeconómicas son $\propto>0 ; \delta>0$ y $\gamma>0$. Anuncios corresponde al mismo conjunto de variables ficticias utilizadas anteriormente (dummy_aum, dummy_red y dummy_neutra). El período de estudio se extiende de junio de 2003 a abril de 2011, y comprende desde la 85a reunión hasta la $158^{a}$ reunión del COPOM.

El análisis empírico se efectuó mediante el método de mínimos cuadrados ordinarios (MCO) ${ }^{12}$ y el método generalizado de momentos (MGM). La razón para usar el método MGM es que mientras las estimaciones mediante MCO presentan problemas de autocorrelación serial, heterocedasticidad o no linealidad, lo que es típico en series temporales macroeconómicas, el método MGM proporciona estimadores consistentes para la regresión (Hansen, 1982).

\footnotetext{
${ }^{9}$ Código 4191 (sitio web del Banco Central del Brasil).

10 Obtenido mediante el filtro de Hodrick-Prescott.

11 Obtenido del sitio web del Banco Central del Brasil.

$12 \mathrm{El}$ estadístico $t$ presentado en las estimaciones mediante MCO se basa en el estimador de Newey y West (1987), que es consistente en presencia tanto de heterocedasticidad como de autocorrelación de forma desconocida.
}

Según Wooldridge (2001, pág. 95), si se procura obtener un estimador más eficiente que los mínimos cuadrados en dos etapas (o mínimos cuadrados ordinarios), se deben tener restricciones fundamentales. Por medio de una prueba $J$ estándar se intentó verificar esta propiedad en cuanto a la validez de las restricciones de superidentificación (Hansen, 1982). Cragg (1983) sostiene que el uso del análisis de superidentificación en la selección de las variables instrumentales mejora la eficiencia de los estimadores. Es necesario que los instrumentos seleccionados estén fechados en el período $t-1$, o antes, para poder predecir las variables contemporáneas, que no están disponibles en el período $t$. Este procedimiento de selección de variables instrumentales está de acuerdo con Johnston (1984) ${ }^{13}$.

Una primera condición a analizar antes de aplicar las estimaciones es verificar si las series son estacionarias. Sobre la base de las pruebas, mientras la serie de expectativas de inflación es I(1), las demás series son I(0) (véanse los cuadros A.2 y A.3 del apéndice).

\section{Resultados}

Los resultados se presentan en los cuadros 2, 3 y 4 . Las estimaciones muestran que para todas las especificaciones, la constante es positiva y estadísticamente significativa, excepto por la ecuación $\left(S_{120}\right.$ a) que no es estadísticamente significativa. En relación con la variable ficticia dummy_break, todos los coeficientes calculados son positivos y estadísticamente significativos, excepto aquellos estimados en las ecuaciones $\left(S_{120} \mathrm{e}\right)$ mediante MCO y $\left(S_{120} \mathrm{~d}\right)$ por medio de MGM, que no son estadísticamente significativos.

Con respecto a las expectativas de inflación, los resultados muestran que todos los coeficientes estimados en el cuadro 2 son positivos, si bien los estimados en las ecuaciones $\left(S_{120} \mathrm{c}\right),\left(S_{120} \mathrm{~d}\right)$ y $\left(S_{120} \mathrm{f}\right)$ mediante MCO y $\left(S_{120} \mathrm{c}\right)$ y $\left(S_{120} \mathrm{f}\right)$ por medio de MGM no son estadísticamente significativos. En el cuadro 3, las estimaciones mediante MCO indican una relación positiva entre las

\footnotetext{
13 En las estimaciones por MGM se aplican las siguientes variables instrumentales:

- Para Swap_120: constante, $S_{-1} 120, S_{-2}{ }^{120}, S_{-3} 120, \mathrm{~d}_{\pi^{2}-2}, \mathrm{~d}_{\pi^{2}-3}, \mathrm{~d}_{\pi^{2}-4}$, $\mathrm{d}_{\pi^{2}-5}, \mathrm{~d}_{\pi^{2}-6}, \mathrm{~d}_{\pi^{2}-7},\left(y-y^{*}\right)_{-2},\left(y-y^{*}\right)_{-3},\left(y-y^{*}\right)_{-4}, \mathrm{y} r_{-2}, r_{-3}, r_{-4}$, $r_{-5}, r_{-6}, r_{-7}, r_{-8}$.

- Para Swap_180: constante, $S_{-1}^{180}, S_{-2}^{180}, S_{-3}^{180}, \mathrm{~d}_{\pi^{2}-2}, \mathrm{~d}_{\pi^{2}-3}, \mathrm{~d}_{\pi^{2}-4}$, $\mathrm{d}_{\pi^{2}-5}, \mathrm{~d}_{\pi^{2}-6}, \mathrm{~d}_{\pi^{2}-7},\left(y-y^{*}\right)_{-2},\left(y-y^{*}\right)_{-3},\left(y-y^{*}\right)_{-4}, \mathrm{y} r_{-2}, r_{-3}, r_{-4}$, $r_{-5}, r_{-6}, r_{-7}, r_{-8}$.

- Para Swap_360: constante, $S_{-1}{ }^{360}, S_{-2}{ }^{360}, S_{-3}{ }^{360}, \mathrm{~d}_{\pi^{2}-2}, \mathrm{~d}_{\pi^{2}-3}, \mathrm{~d}_{\pi^{2}-4}$, $\mathrm{d}_{\pi^{2}-5}, \mathrm{~d}_{\pi^{2}-6}, \mathrm{~d}_{\pi^{2}-7},\left(y-y^{*}\right)_{-2},\left(y-y^{*}\right)_{-3},\left(y-y^{*}\right)_{-4}, \mathrm{y} r_{-2}, r_{-3}, r_{-4}$, $r_{-5}, r_{-6}, r_{-7}, r_{-8}$.
} 
expectativas de inflación y la variable $S w a p \_180$, si bien no es estadísticamente significativa en la ecuación $\left(S_{180} \mathrm{~d}\right)$. Con el método MGM, los coeficientes estimados en las ecuaciones $\left(S_{180} \mathrm{a}\right),\left(S_{180} \mathrm{~b}\right),\left(S_{120} \mathrm{~d}\right)$, $\left(S_{120} \mathrm{e}\right)$ y $\left(S_{120} \mathrm{f}\right)$ son todos positivos y estadísticamente significativos. Los resultados correspondientes a la variable Swap_360 muestran coeficientes positivos y estadísticamente significativos en todas las especificaciones de ambos métodos (véase el cuadro 4). La explicación económica para la relación positiva entre las expectativas de inflación y las tasas a corto plazo es que la esperanza estadística de una mayor inflación genera expectativas de que el banco central ajustará la política monetaria mediante el aumento de las tasas de interés a corto plazo en el futuro, para desacelerar el crecimiento económico y contener las presiones inflacionarias. Asimismo, las expectativas de inflación repercuten con fuerza en las tasas de interés a más largo plazo del tramo corto de la estructura temporal. Esto se debe, básicamente, a que las expectativas de inflación son las expectativas del mercado para los próximos 12 meses.

Las estimaciones que se presentan en los cuadros 2, 3 y 4 también muestran la influencia positiva de las desviaciones del producto en las tasas a corto plazo. Todas las especificaciones en el cuadro 2 son estadísticamente significativas, mientras que en los cuadros 3 y 4 las especificaciones $\left(S_{180}\right.$ a), $\left(S_{360}\right.$ a) y $\left(S_{360}\right.$ c), tanto mediante MCo como por medio del método MGM, no son estadísticamente significativas. La relación positiva entre las desviaciones del producto (una medida del ciclo económico) y las tasas a corto plazo se explica de la siguiente manera: cuando la economía se agiliza aumentan las presiones inflacionarias, lo que genera que los agentes económicos prevean una subida de las tasas de interés en el futuro.
La política monetaria tiene una relación directa con el tramo corto de la estructura temporal, es decir, cuando la tasa SELIC aumenta, las tasas swap también suben. Sobre la base de los valores de los coeficientes estimados, este efecto es menor en las tasas de interés a más largo plazo. En este sentido, la política monetaria del Banco Central del Brasil, a través de la tasa SELIC, ejerce una gran influencia en el tramo corto de la estructura temporal.

Los anuncios del banco central también tienen un efecto positivo en las tasas a corto plazo. La variable ficticia para una señal alcista, dummy_aum, tiene un efecto positivo estadísticamente significativo en el tramo corto de la estructura temporal. En otras palabras, cuando las actas del Cором revelan una posible subida de la tasa de interés SELIC en la próxima reunión, las tasas de interés a corto plazo, medidas por las tasas swap, aumentan. Una comparación entre las variables Swap_120 y Swap_360 sugiere que los anuncios que indican políticas monetarias de ajuste en el futuro tienen un efecto más pronunciado en la tasa swap a 360 días que en la tasa swap a 120 días.

Para una señal a la baja, los resultados correspondientes a la variable ficticia dummy_red revelan una repercusión negativa estadísticamente significativa en el tramo corto de la estructura temporal. Cuando el COPOM brasileño indica un posible descenso de la tasa SELIC en la próxima reunión, las tasas de interés, medidas por las tasas swap, bajan. Una vez más, los resultados sugieren que los anuncios tienen un efecto mayor en la tasa a 360 días que en la tasa a 120 días.

Ninguno de los coeficientes estimados para la variable ficticia dummy_neutra es estadísticamente significativo. Esto significa que cuando el COPOM señala que no cambiará la tasa de interés SELIC en la próxima reunión, las tasas de interés, medidas por las tasas swap, tampoco cambian. 


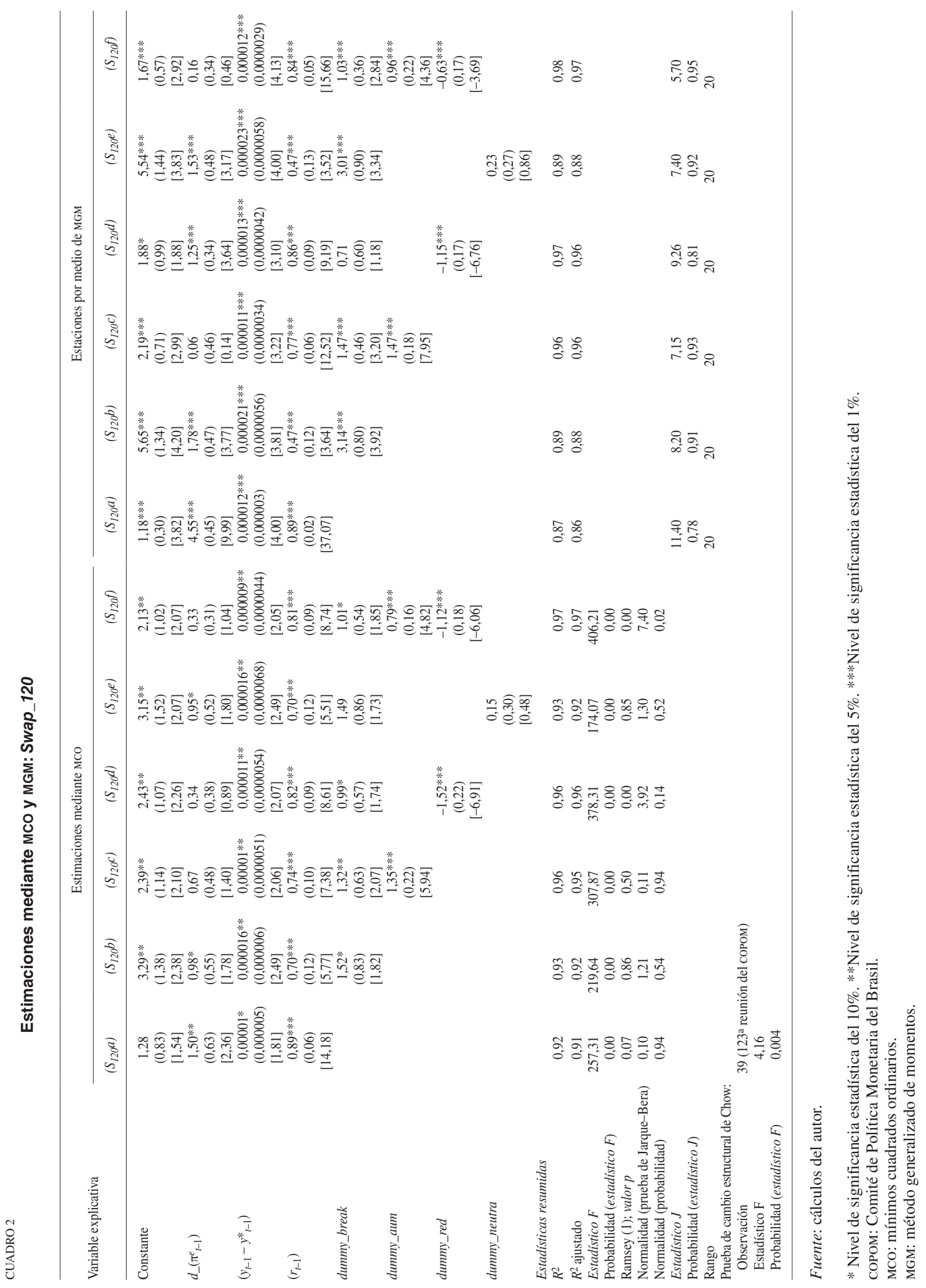




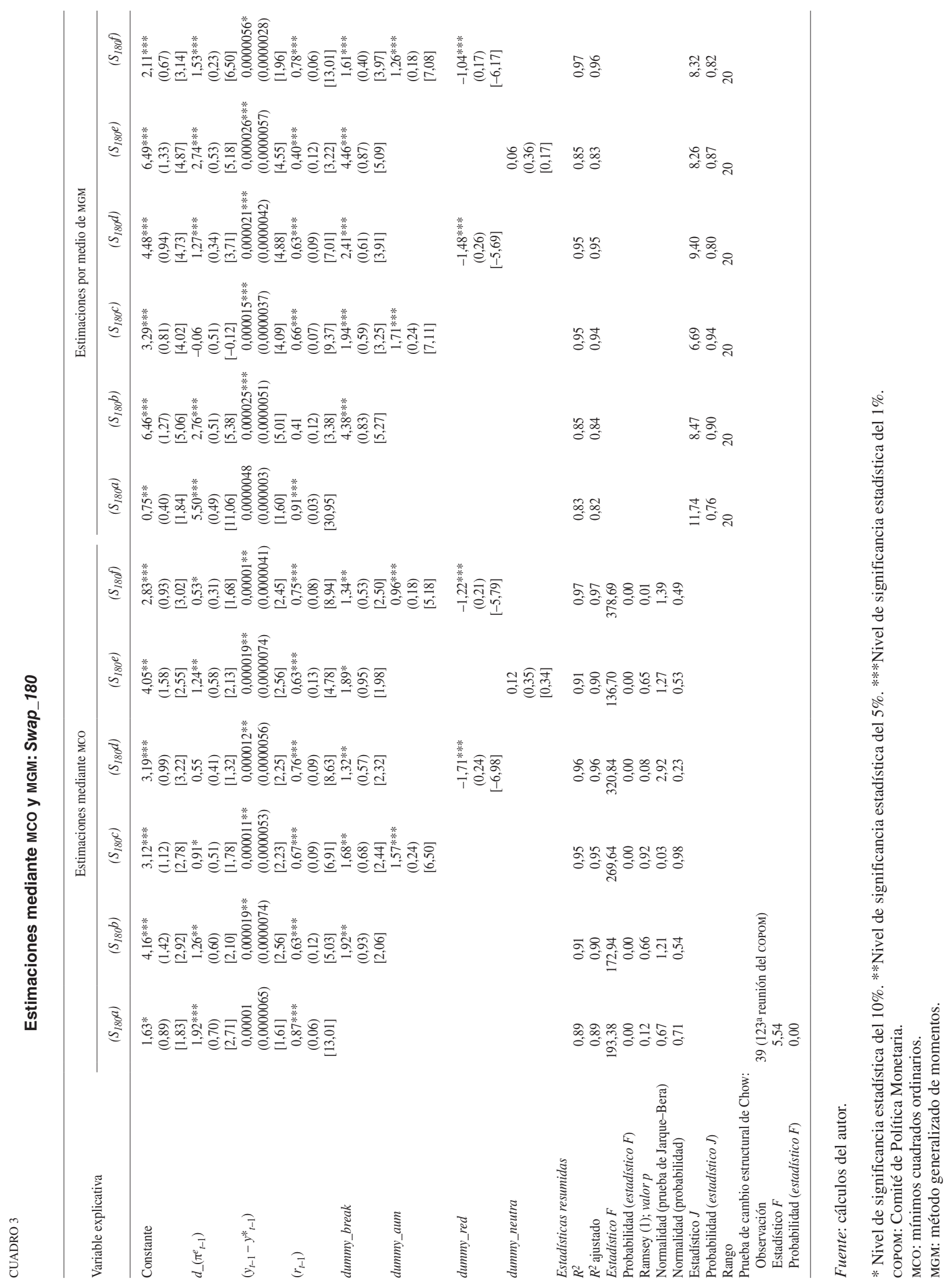




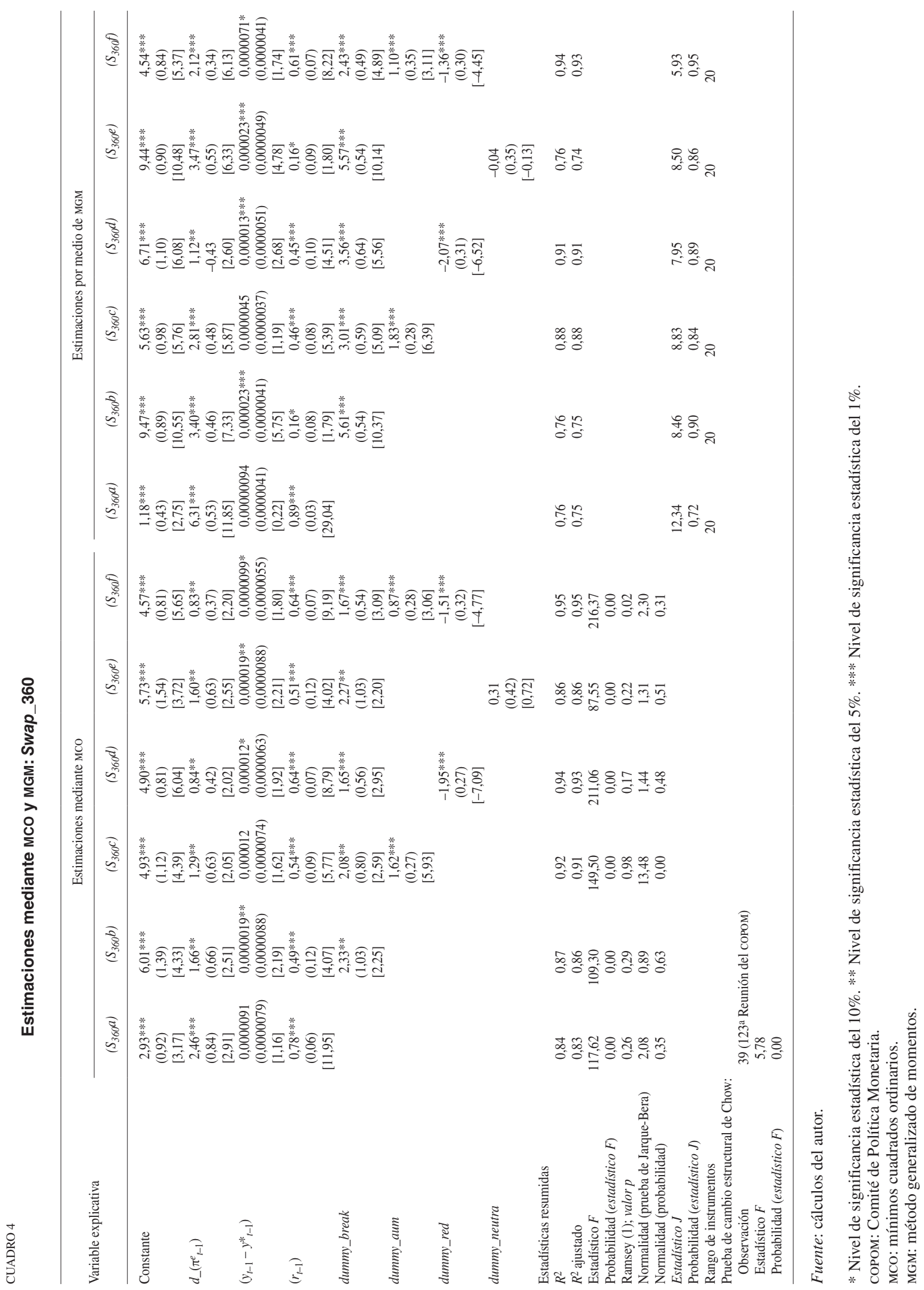




\section{IV}

\section{Conclusiones}

A través de los anuncios, el Banco Central del Brasil tiene la posibilidad de explicar sus intenciones, decisiones y fundamentos. Dado que la fijación de objetivos de inflación permite cierto margen de discreción, los anuncios se convierten en una importante herramienta que permite a los encargados locales de la formulación de políticas explicar sus decisiones y ayudar a los agentes económicos a comprender la percepción del Banco Central del Brasil sobre el estado de la economía y las perspectivas económicas. De ese modo, dichos agentes pueden formar mejor sus expectativas, lo que tiene un efecto directo en las variables de los mercados financieros.

A fin de hacer un aporte a los estudios inherentes al efecto de los anuncios del banco central y la política monetaria en los mercados financieros, en el presente artículo se ha analizado la influencia de estos factores en la estructura temporal de las tasas de interés en el Brasil. En el estudio se demuestra que las acciones de política monetaria y los anuncios por parte de la autoridad monetaria brasileña repercuten intensamente en el proceso de formación de expectativas respecto de las tasas de interés a distintos plazos en el país. Los mercados financieros no solo responden a la publicación de las actas del сором, sino que se mueven en la dirección deseada. En este sentido, las actas de las reuniones del COPOM actúan como una herramienta relevante para guiar las expectativas con relación a las tasas de interés, dado que los agentes económicos utilizan dichas actas para predecir el estado venidero de la economía, lo que reduce la incertidumbre acerca de las acciones futuras en materia de política monetaria.

Los resultados subrayan los efectos beneficiosos de los anuncios en el suministro de información al público en general y a los mercados en particular, configurándose como un importante instrumento del banco central. Sobre la base de la evidencia aportada en este trabajo, la relevancia de los anuncios de la autoridad monetaria radica en su capacidad para afectar las expectativas respecto de las tasas de interés futuras, lo que a su vez sirve de base para la toma de variadas decisiones esenciales de los agentes económicos. Esto es así debido a que las decisiones de política monetaria afectan a las tasas de interés a corto plazo, mientras que las variables relevantes para la política monetaria se ven influenciadas por las tasas de interés a largo plazo. Dado que estas tasas de interés son determinadas por las expectativas respecto de la evolución futura de las tasas de interés, la importancia de los anuncios del banco central estriba en su capacidad de afectar a las expectativas del mercado.

Los anuncios del banco central no son la única variable que determina el proceso de formación de expectativas en los mercados financieros, donde también influyen otras variables. Por consiguiente, además de explorar la repercusión de la política monetaria y los anuncios en las expectativas respecto de las tasas de interés, en el estudio también se analizaron los efectos de las variables macroeconómicas, como son las fluctuaciones del producto y las expectativas de inflación. En investigaciones por hacerse se podría ampliar el modelo a objeto de considerar otras políticas económicas, como la política fiscal, mediante la incorporación de variables, tales como la relación deuda a PIB, el perfil de la deuda y la credibilidad fiscal.

La reacción del mercado financiero a la política monetaria y los anuncios del banco central es sin duda un tema de gran interés para los participantes del mercado y los bancos centrales. Los inversores tienen interés en anticipar las decisiones del banco central dado que estas pueden afectar al valor de sus carteras, mientras que el banco central procura estimar el posible efecto de la política monetaria en los precios de los activos y en la economía en conjunto. En investigaciones futuras se podrían incorporar en forma productiva no solo los temas sugeridos por Blinder y otros (2008), sino también la influencia de los anuncios del banco central en los mecanismos de transmisión de la política monetaria mediante los siguientes canales: i) los precios de los activos financieros y, por lo tanto, las decisiones de consumo e inversión a través del efecto riqueza; ii) el crédito, a través de las posturas de los bancos y las decisiones sobre el crédito; y iii) las expectativas acerca de variables macroeconómicas, como las expectativas de inflación, el tipo de cambio y el crecimiento del producto. 
APÉNDICE

\section{Cuadros y gráficos complementarios}

CUADRO A.1

Brasil: Glosario de términos y expresiones clave de las actas de las reuniones del Comité de Política Monetaria (COPOM)

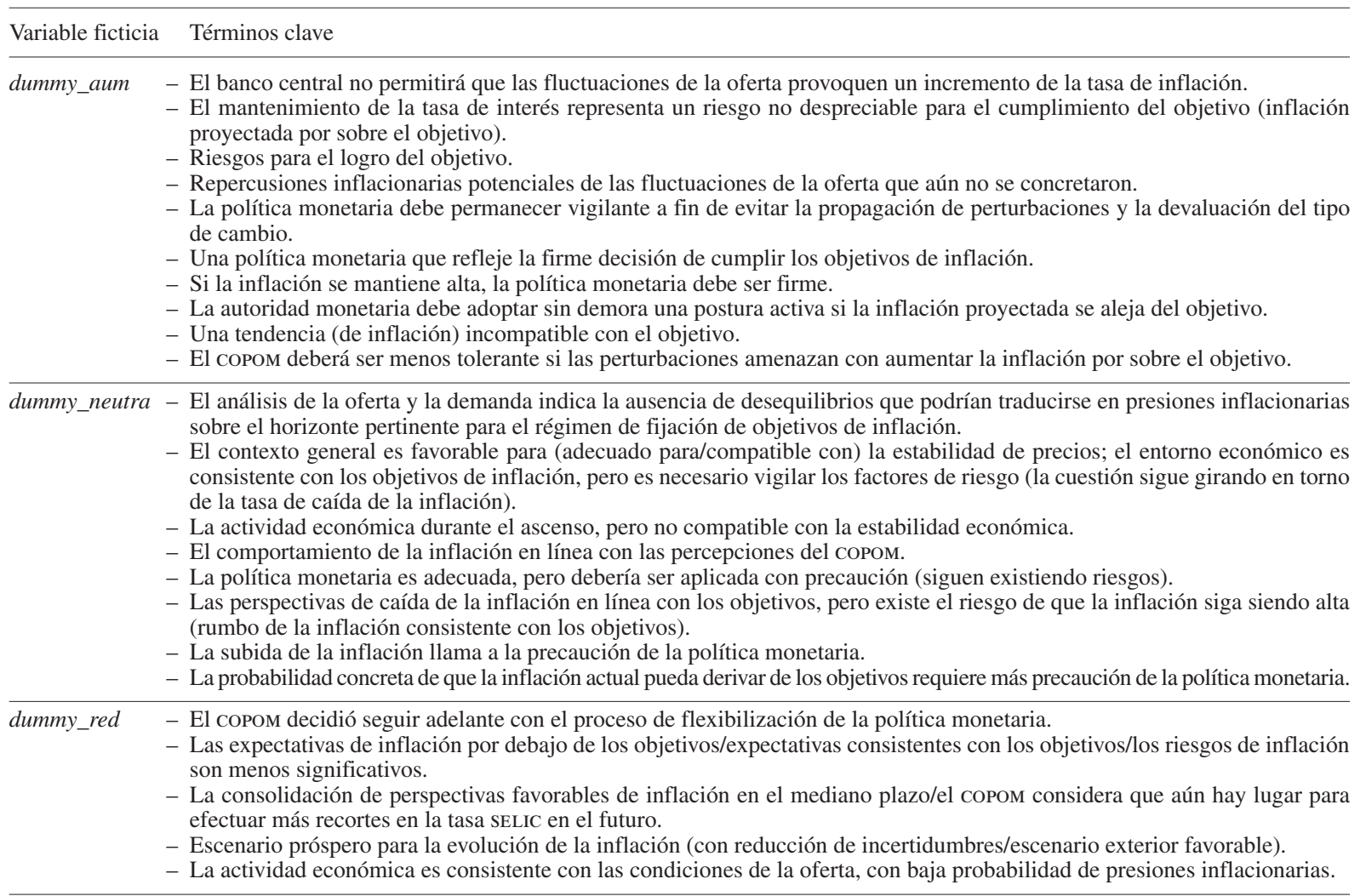

Fuente: elaboración propia.

COPOM: Comité de Política Monetaria.

SELIC: Sistema Especial de Liquidación y Custodia.

CUADRO A.2

Prueba de estacionariedad de Kwiatkowski-Phillips-Schmidt-Shin (KPSS)

\begin{tabular}{lcccc}
\hline Series & Ancho de banda & Prueba & Valores críticos al 1\% & Valores críticos al 5\% \\
\hline Swap_120 & 6 & $0,08^{\mathrm{b}}$ & 0,21 & 0,14 \\
Swap_180 & 6 & $0,08^{\mathrm{b}}$ & 0,21 & 0,14 \\
Swap_360 & 6 & $0,08^{\mathrm{b}}$ & 0,21 & 0,14 \\
Diferencial $_{120}$ & 6 & $0,07^{\mathrm{b}}$ & 0,21 & 0,14 \\
Diferencial $_{180}$ & 6 & $0,07^{\mathrm{b}}$ & 0,21 & 0,11 \\
Diferencial $_{360}$ & 6 & $0,07^{\mathrm{b}}$ & 0,21 & 0,14 \\
Expectativa de inflación & 6 & $0,22^{\mathrm{b}}$ & 0,21 & 0,11 \\
d(expectativa de inflación) & 2 & $0,04^{\mathrm{b}}$ & 0,21 & 0,11 \\
Brecha de producción & 5 & $0,03^{\mathrm{a}}$ & 0,73 & 0,11 \\
Tasa SELIC & 6 & $0,06^{\mathrm{b}}$ & 0,21 & 0,11 \\
\hline
\end{tabular}

Fuente: cálculos propios.

Nota: se utilizó el método de selección de ancho de banda de Andrews y el núcleo (kernel) de Bartlett. Se empleó el criterio de información de Schwarz.

SELIC: Sistema Especial de Liquidación y Custodia.

a Indica constante.

b Indica constante y tendencia. 
CUADRO A.3

Prueba de raíz unitaria de Phillips-Perron (PP)

\begin{tabular}{lcccc}
\hline Series & Ancho de banda & Prueba & Valores críticos al 1\% & Valores críticos al 5\% \\
\hline Swap_120 & 6,51 & $-1,738^{\mathrm{c}}$ & $-2,597$ & $-1,945$ \\
Swap_180 & 5,99 & $-1,654^{\mathrm{c}}$ & $-2,597$ & $-1,945$ \\
Swap_360 $_{\text {Diferencial }}$ 120 & 2,51 & $-1,614^{\mathrm{c}}$ & $-2,597$ & $-1,945$ \\
Diferencial $_{180}$ & 1,70 & $-3,647^{\mathrm{c}}$ & $-2,597$ & $-1,945$ \\
Diferencial $_{360}$ & 1,37 & $-3,525^{\mathrm{c}}$ & $-2,597$ & $-1,945$ \\
Expectativa de inflación $_{\text {(expectativa de inflación) }}$ & 0,08 & $-3,416^{\mathrm{c}}$ & $-2,597$ & $-1,945$ \\
Brecha de producción & 3,06 & $-2,378^{\mathrm{a}}$ & $-3,522$ & $-2,901$ \\
Tasa SELic & 0,72 & $-7,150^{\mathrm{c}}$ & $-2,597$ & $-1,945$ \\
\hline
\end{tabular}

Fuente: cálculos propios.

Nota: se utilizó el método de selección de ancho de banda de Andrews y el núcleo (kernel) de Bartlett. Se utilizó el criterio de información de Schwarz.

SELIC: Sistema Especial de Liquidación y Custodia.

a Indica constante.

b Indica constante y tendencia.

c indica su ausencia.

CUADRO A. 4

Criterios de AIC, SIC y HQ para el orden de rezago del modelo VAR

\begin{tabular}{|c|c|c|c|c|c|c|}
\hline \multirow{2}{*}{ Orden de retardo del VAR } & \multicolumn{3}{|c|}{ Con constante } & \multicolumn{3}{|c|}{ Sin constante } \\
\hline & AIC & SIC & HQ & AIC & SIC & HQ \\
\hline 0 & 7,74 & 7,94 & 7,82 & & & \\
\hline 1 & $-0,13$ & $1,23 *$ & $0,40 *$ & $-0,03 *$ & $1,13^{*}$ & $0,42 *$ \\
\hline 2 & 0,14 & 2,68 & 1,15 & 0,25 & 2,60 & 1,18 \\
\hline 3 & 0,29 & 4,01 & 1,77 & 0,49 & 4,01 & 1,88 \\
\hline 4 & 0,53 & 5,42 & 2,47 & 0,82 & 5,52 & 2,68 \\
\hline 5 & 0,27 & 6,34 & 2,67 & 0,57 & 6,44 & 2,89 \\
\hline 6 & $-0,24$ & 7,00 & 2,62 & 0,01 & 7,06 & 2,81 \\
\hline
\end{tabular}

Fuente: cálculos propios.

Notas: criterio de información de Akaike (AIC); criterio de información de Schwarz (sIC); criterio de información de Hannan-Quinn (HQ).

Los asteriscos indican el orden de retardo seleccionado por el criterio respectivo. 
GRÁFICO A.1

\section{Corte estructural: Swap_120}

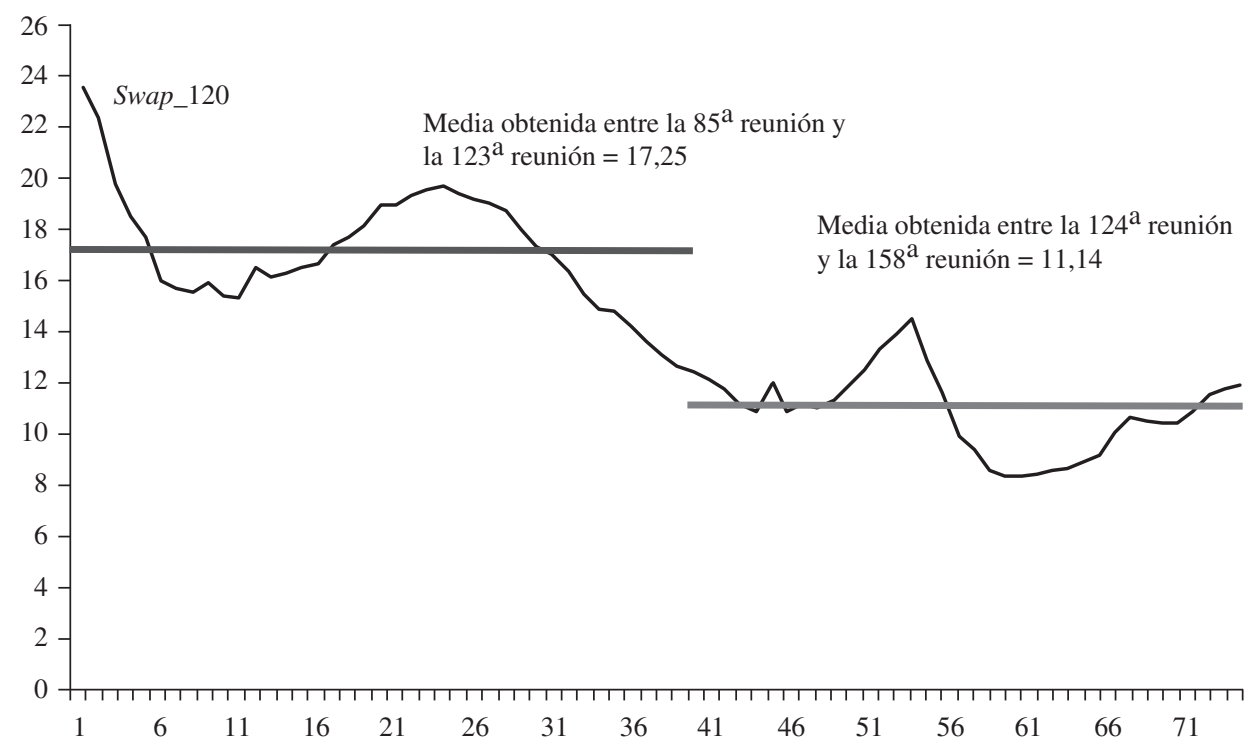

Fuente: elaboración propia.

GRÁFICO A.2

\section{Corte estructural: Swap_180}

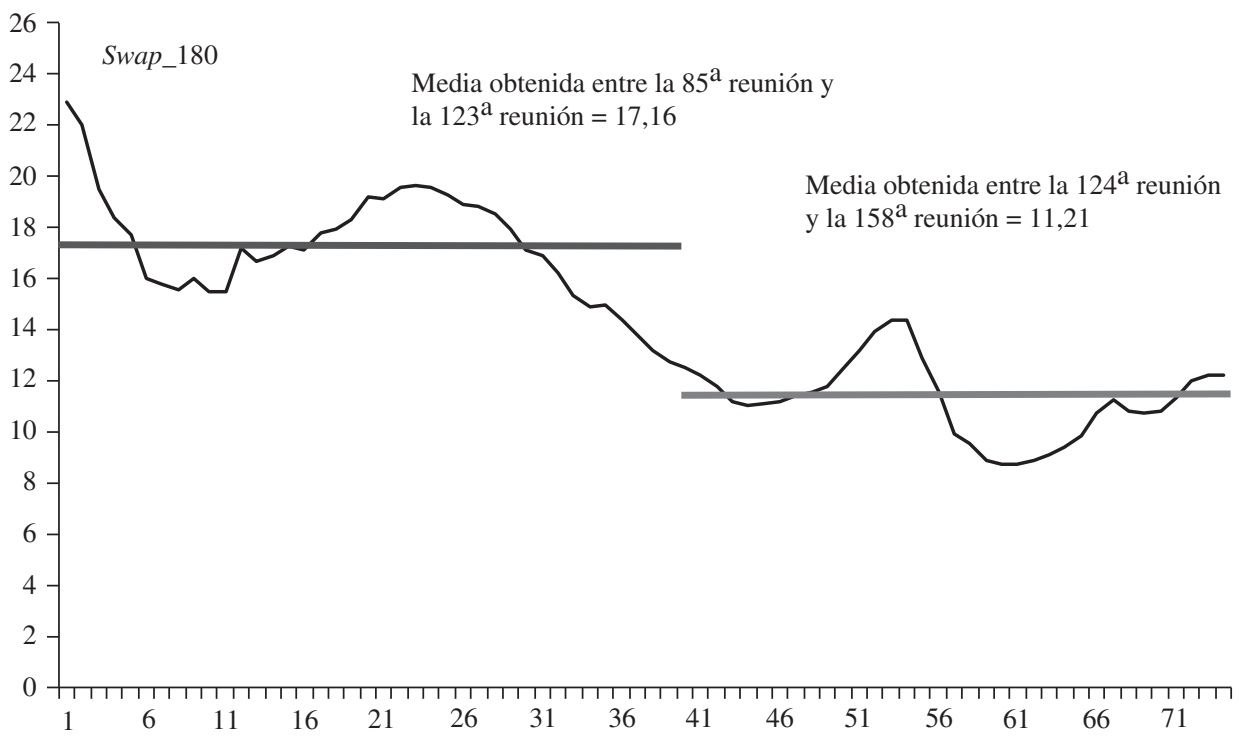

Fuente: elaboración propia. 


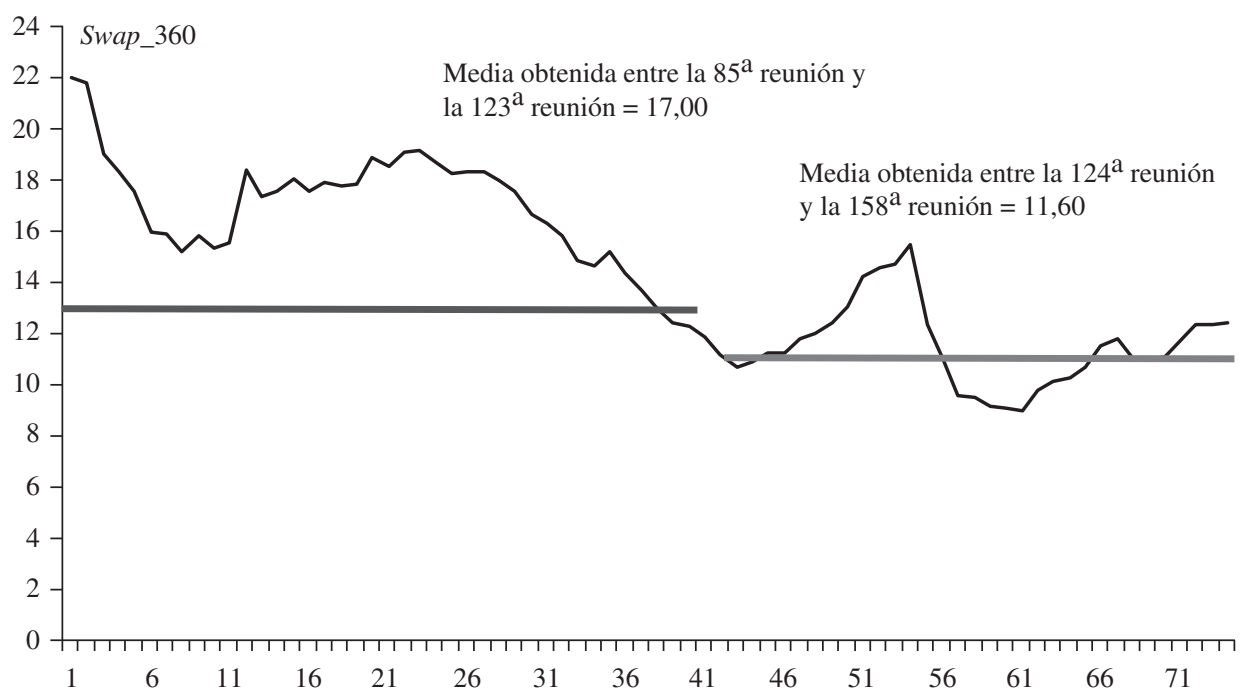

Fuente: elaboración propia.

Andersson, M., H. Dillén y P. Sellin (2006), "Monetary policy signaling and movements in the Swedish term structure of interest rates", Journal of Monetary Economics, vol. 53, $\mathrm{N}^{\circ} 8$, Amsterdam, Elsevier.

Berger, H., J. de Haan y J-E. Sturm (2011), "Does money matter in the ECB strategy? New evidence based on ЕСв communication", International Journal of Economics and Finance, vol. 16, $\mathrm{N}^{\mathrm{o}} 1$, John Wiley \& Sons.

Blinder, A. y otros (2008), "Central Bank communication and monetary policy: a survey of theory and policy", Journal of Economic Literature, vol. 46, $\mathrm{N}^{\circ}$ 4, Nashville, Tennessee, American Economic Association.

Brand, C., D. Buncic y J. Turunen (2010), "The impact of ECB monetary policy decisions and communication on the yield curve", Journal of the European Economic Association, vol. 8, № 6, Cambridge, Massachusetts, The MIT Press.

Connolly, E. y M. Kohler (2004), "News and interest rate expectations: a study of six central banks", RBA Research Discussion Paper, 04-10, Sydney, Reserve Bank of Australia.

Cook, T. y T. Hahn (1989), "The effect of changes in the federal funds rate target on market interest rates in the 1970s", Journal of Monetary Economics, vol. 24, No 3, Amsterdam, Elsevier.

Costa Filho, A.E. y F. Rocha (2010), "Como o mercado de juros futuro reage a comunicação do banco central?", Economia aplicada, vol. $14, \mathrm{~N}^{\circ} 3$.

(2009), "Comunicação e política monetária no el Brasil", Revista brasileira de economia, vol. 63, № 4, Río de Janeiro, Fundación Getulio Vargas.

Cragg, J.G. (1983), "More efficient estimation in the presence of heteroscedasticity of unknown form", Econometrica, vol. 51, $\mathrm{N}^{\circ} 3$, Nueva York, Econometric Society.

De Mendonça, H.F. y J. Simão-Filho (2007), "Economic transparency and effectiveness of monetary policy", Journal of Economic Studies, vol. 34, N 6, Emerald Group Publishing.
Ehrmann, M. y M. Fratzscher (2009), "Explaining monetary policy decisions in press conferences", International Journal of Central Banking, vol. 5, No 2, San Francisco.

(2007a), "The timing of central bank communication", European Journal of Political Economy, vol. 23, № 1, Amsterdam, Elsevier. (2007b), "Communication by Central Bank Committee members: different strategies, same effectiveness", Journal of Money, Credit and Banking, vol. 39, No 2-3, Blackwell Publishing.

Evans, C.L. y D.A. Marshall (1998), "Monetary policy and the term structure of nominal interest rates: evidence and theory", Carnegie-Rochester Conference Series on Public Policy, vol. 49, $\mathrm{N}^{\mathrm{o}}$ 1, Amsterdam, Elsevier.

Ewing, B.T. (2003), "The response of the default risk premium to macroeconomic shocks", The Quarterly Review of Economics and Finance, vol. 43, $\mathrm{N}^{\circ} 2$, Amsterdam, Elsevier.

Fuhrer, J.C. (1996), "Monetary policy shifts and long term interest rates", Quarterly Journal of Economics, vol. 111, No 4, Cambridge, Massachusetts, The MIT Press.

Fuhrer, J.C. y G.R. Moore (1995a), "Inflation persistence", Quarterly Journal of Economics, vol. 110, $\mathrm{N}^{\circ}$ 1, Cambridge, Massachusetts, The MIT Press.

(1995b), "Monetary policy trade-offs and the correlation between nominal interest rates and real output", The American Economic Review, vol. 85, № 1, Nashville, Tennessee, American Economic Association.

Fujiwara, I. (2005), "Is the Central Bank's publication of economic forecasts influential?", Economics Letters, vol. 89, No 3 , Amsterdam, Elsevier.

Geraats, P. (2002), "Central Bank transparency", The Economic Journal, vol. $112, \mathrm{~N}^{\circ} 483$, Wiley.

Haldane, A.G. y V. Read (2000), "Monetary policy surprises and the yield curve", Bank of England Working Paper, № 106, Londres, Bank of England. 
Hansen, L.P. (1982), "Large sample properties of generalized method of moments estimators", Econometrica, vol. 50, No 4, Nueva York, Econometric Society.

Hayo, B., A. Kutan y M. Neuenkirch (2010), "The impact of U.S. central bank communication on European and pacific equity markets", Economics Letters, vol. 108, No 2, Amsterdam, Elsevier.

Hayo, B. y M. Neuenkirch (2010), "Do Federal Reserve communications help predict federal funds target rate decisions?", Journal of Macroeconomics, vol. 32, $\mathrm{N}^{\circ}$ 4, Amsterdam, Elsevier.

Jansen, D. y J. de Haan (2005), "Talking heads: the effects of ЕСB statements on the euro-dollar exchange rate", Journal of International Money and Finance, vol. 24, $\mathrm{N}^{\circ} 2$, Amsterdam, Elsevier.

Johnston, J. (1984), Econometric Methods, Singapur, McGraw-Hill.

Kohn, D.L. y B. Sack (2004), "Central bank talk: does it matter and why?", Macroeconomics, Monetary Policy, and Financial Stability, Ottawa, Banco de Canadá.

Koop, G., M.H. Pesaran y S.M. Potter (1996), "Impulse response analysis in non-linear multivariate models", Journal of Econometrics, vol. 74, $\mathrm{N}^{\mathrm{o}} 1$, Amsterdam, Elsevier.

Lima, A. y J. Issler (2003), "A hipótese das expectativas na estrutura a termo de juros no el Brasil: uma aplicação de modelos de valor presente", Revista brasileira de economia, vol. 57, № 4, Río de Janeiro, Fundación Getulio Vargas.

Lutkenpohl, H. (1991), Introduction to Multiple Time Series Analysis, Berlín, Springer.

Mishkin, F.S. y M.A. Savastano (2001), "Monetary policy strategies for Latin America", Journal of Development Economics, vol. 66, $\mathrm{N}^{\mathrm{o}}$ 2, Amsterdam, Elsevier.

Montes, G.C. y J.C.A. Bastos (2011), "Metas de inflação e estrutura a termo das taxas de juros no el Brasil", Economia aplicada, vol. 15, No 3, São Paulo.

Musard-Gies, M. (2006), "Do ECB's statements steer short-term and long-term interest rates in the Euro-Zone?", The Manchester School, vol. 74 (supplement), Wiley.

Newey, W.K. y K.D. West (1987), "A simple, positive semidefinite, heteroskedasticity and autocorrelation consistent covariance matrix", Econometrica, vol. 55, No 3, Nueva York, Econometric Society.
Pesaran, M.H. e Y. Shin (1998), "Generalized impulse response analysis in linear multivariate models", Economics Letters, vol. 58, $\mathrm{N}^{\mathrm{o}} 1$, Amsterdam, Elsevier.

Ranaldo, A. y E. Rossi (2010), "The reaction of asset markets to Swiss National Bank communication", Journal of International Money and Finance, vol. 29, $\mathrm{N}^{\circ} 3$, Amsterdam, Elsevier.

Reeves, R. y M. Sawicki (2007), "Do financial markets react to Bank of England communication?", European Journal of Political Economy, vol. 23, № 1, Amsterdam, Elsevier.

Roley, V. y G. Sellon (1995), "Monetary policy actions and long term interest rates", Economic Review, cuarto trimestre, Kansas, Federal Reserve Bank of Kansas City.

Rosa, C. (2011), "Talking less and moving the market more: evidence from the ECB and the Fed", Scottish Journal of Political Economy, vol. $58, \mathrm{~N}^{\mathrm{o}} 1$,Wiley.

Rosa, C. y G. Verga (2007), "On the consistency and effectiveness of Central Bank communication: evidence from the ЕСв", European Journal of Political Economy, vol. 23, $\mathrm{N}^{\circ} 1$, Amsterdam, Elsevier.

Rozkrut, M. y otros (2007), "Quest for Central Bank communication. Does it pay to be "talkative?", European Journal of Political Economy, vol. 23, № 1, Amsterdam, Elsevier.

Sims, C. (1980), "Macroeconomics and reality", Econometrica, vol. 48, $\mathrm{N}^{\circ} 1$, Nueva York, Econometric Society.

Sturm, J.-E. y J. De Haan (2011), "Does central bank communication really lead to better forecasts of policy decisions? New evidence based on a Taylor rule model for the ECB", Review of World Economics, vol. 147, $\mathrm{N}^{\circ} 1$, Springer.

Tabak, B.M. y A. Tabata (2004), "Surpresas na política monetária e a estrutura a termo da taxa de juros brasileira", Revista de economia aplicada, vol. $8, \mathrm{~N}^{\circ} 3$.

Walsh, C.E. (2010), Monetary Theory and Policy, Cambridge, Massachusetts, The MIT Press.

Woodford, M. (2003), Interest and Prices: Foundations of a Theory of Monetary Policy, Princeton, Princeton University Press.

Wooldridge, J.M. (2001), "Applications of generalized method of moments estimation", Journal of Economic Perspectives, vol. 15, No 4, Nashville, Tennessee, American Economic Association. 